\title{
Liquid-Crystal alignment by a nanoimprinted grating for wafer-scale fabrication of tunable devices
}

\author{
B. Sadani, B. Boisnard, X. Lafosse, T. Camps, J.B. Doucet, E. Daran, C. Paranthoen, C. Levallois, L. \\ Dupont, S. Bouchoule, and V. Bardinal
}

\begin{abstract}
A simple technology is demonstrated for waferscale fabrication of liquid-crystal (LC) microcells that can be integrated in active optoelectronic devices. Fabrication of $1.55 \mu \mathrm{m}$ tunable Fabry-Perot optical micro-filter arrays is achieved owing to the insertion of a single nanoimprinted polymer grating dedicated to $L C$ alignment and to the soft thermal transfer of a dry thick resist film between two highly reflective mirrors. The filter exhibits a spectral tuning range of $102 \mathrm{~nm}$ with only $18 \mathrm{~V}$ applied, as well as negligible internal loss, which makes it suitable for being inserted in a laser cavity. This constitutes a key step toward large-scale integration of widely-tunable photonic devices such as VCSELs using LC technology.
\end{abstract}

Index Terms-liquid crystal devices, tunable optical filter, wafer-scale fabrication, nanoimprint, grating, dry thick resist film, soft transfer, SU-8, VCSEL.

\section{INTRODUCTION}

T UNABLE photonic devices are of great interest for the development of low cost optical communications systems and compact sensors. In particular, CWDM applications require $1.55 \mu \mathrm{m}$ devices such as photodetectors or VCSELs (verticalcavity surface-emitting lasers), that can be tuned over a wide spectral range with low electrical consumption and no mode hopping. To date, available devices are based on the modification of micro-cavity length by actuation of a movable mirror [1]. An alternative to this "MEMS-based" approach could be the integration of a birefringent liquid crystal (LC) intracavity layer. In this way, it would be possible to fabricate monolithic tunable devices without any movable part. Many

Copyright (c) 2018 IEEE. Submission date: 27 March 2018

The authors acknowledge RENATECH (French Network of Major Technology Centers) within LAAS-CNRS, C2N-CNRS/UPSUD and Nanorennes for technological support. The French National Research Agency (ANR) is acknowledged for financial support (ANR-14-ASTR-0007 HYPOCAMP and ANR-15-CE19-0012 DOCT VCSEL). The authors acknowledge also R.-M. Sauvage (DGA/DS/MRIS) and Y. Leger (FOTON) for fruitful discussions and technical support.

B. Sadani, B. Boisnard, J.B. Doucet, E. Daran and V. Bardinal are with LAAS-CNRS, Université de Toulouse, CNRS, 7 avenue du colonel Roche, F31400 Toulouse, France. T. Camps is with LAAS-CNRS, Université de Toulouse, CNRS, UPS, 7 avenue du colonel Roche, F-31400 Toulouse, France. (e-mail: bardinal@laas.fr). demonstrations of passive LC-based tunable Fabry-Perot (FP) interferometers filters were reported in the 1990s [2]-[3]-[4]. However, despite a renewed interest for active LC-based photonic devices [5]-[6]-[7] and the demonstration of an optically-pumped tunable LC-VCSEL in 2011 [8], the fabrication of electrically driven tunable active devices has not been demonstrated yet, as this implies to combine LC alignment with electrode management at a micro-scale size. Alignment of liquid crystal molecules is indeed mandatory in these polarization-sensitive devices. Generally, it is ensured by the creation of anisotropy on one or on both surface(s) in contact with the LC material. Polyimide surface rubbing is the most used method, especially for the fabrication of large-area Liquid Crystal Displays (LCDs), as it is very simple, parallel and costeffective. However, this approach is not compatible with microscale device fabrication and non-planar surface topography. Another solution consists in the use of photo-alignment layers [9]. Although collective fabrication of LC microcells using such alignment films was reported [10], this method is not easy to apply in a multi-layer process. Alternatively, LC alignment can be obtained through a planar anchoring on a topological pattern such as a surface relief grating [11]. In this case, the LC preferential orientation is along the grating grooves, as this configuration minimizes the LC elastic deformation energy. A macro-scale resonant FP filter including two nano-gratings fabricated by NIL (Nano-imprint Lithography) was reported with a tuning range of $20 \mathrm{~nm}$ and a $1.5 \mathrm{~nm}$ linewidth [12]. Nonetheless, the association of nano-gratings to micro-cells arrays is not yet demonstrated at the wafer-scale. In the present study, LC-tunable FP filter arrays including a single nanoimprinted grating and polymer microcells are for the first time fabricated with low optical losses and a tuning range of $\sim 100$

S. Bouchoule and X. Lafosse are with $\mathrm{C} 2 \mathrm{~N}$ (Centre for Nanoscience and nanotechnology), CNRS, Université Paris-Sud, Route de Nozay, 91460 Marcoussis, France.(e-mail: sophie.bouchoule@c2n.upsaclay.fr).

L. Dupont is with IMT Atlantique, Optics Department, 655 Avenue du Technopôle, 29200 Plouzané, France. (e-mail: laurent.dupont@imtatlantique.fr).

C. Paranthoen and C. Levallois are with Univ Rennes, INSA Rennes, CNRS, Institut FOTON - UMR 6082, F-35000 Rennes, France. (e-mail: Cyril.Paranthoen@insa-rennes.fr). 
$\mathrm{nm}$ at $1.55 \mu \mathrm{m}$. Furthermore, this demonstrator exhibits a $0.35 \mathrm{~nm}$ linewidth and is fully compatible with future waferscale integration in a VCSEL device.

\section{DESIGN AND TECHNOLOGY}

\section{A. LC-microcell tunable narrow-band filter design}

For a future integration with an active device such as a VCSEL, the LC microcell technology should induce negligible optical losses. A high-quality factor Fabry-Perot filter was therefore designed to evaluate the residual losses. The schematic cross-sectional view of the tunable micro-filter is shown in Fig. 1a. It is composed of two dielectric distributed Bragg reflectors (DBRs) deposited on top of a transparent conductive electrode (ITO, 20nm thick and $\mathrm{R} / \square \sim 100 \Omega$ ) on 2 inches glass substrates with a bilayer $\left(\mathrm{TiO}_{2} / \mathrm{SiO}_{2}\right)$ antireflection coating (AR) on the backside. Each DBR is composed of $8 \mathrm{x}\left(\mathrm{SiO}_{2} / \mathrm{TiO}_{2}\right)$ periods corresponding to a nominal reflectivity of $99.7 \%$ at $1.55 \mu \mathrm{m}$ and a stop-band width (at half-maximum) of $\sim 500 \mathrm{~nm}$. After the definition of $5-\mu \mathrm{m}$ high microcells and subsequent LC infiltration (see section C, and Fig. 1(a)), the microcell acts as a birefringent filter. The incident light with a linear polarization perpendicular to the grating grooves experiences a fixed refractive index in the LC medium, $\mathrm{n}_{0}$ (ordinary mode). In case of planar anchoring and without any applied voltage $(\mathrm{V}=0)$, the incident light with a linear polarization parallel to the grating grooves experiences a refractive index equal to the extraordinary index $n_{e}$ (see Fig.1(b)). When applying a voltage and above a threshold, this refractive index decreases until it reaches the ordinary index value $\mathrm{n}_{\mathrm{o}}$ [13]. The resonant mode with a linear polarization parallel to the grating grooves will be referred to as "extraordinary mode" in the following. In this work, we used a commercial nematic LC (E7), presenting a large birefringence $(\Delta \mathrm{n}=0.19)$ at $1.55 \mu \mathrm{m}$ on the extraordinary mode [13][14] with a nematic temperature range from $-20^{\circ} \mathrm{C}$ to $70{ }^{\circ} \mathrm{C}$. In a simple design, both the bottom and top electrodes correspond to common electrodes for all the micro-cells. In an advanced design, each microcell electrodes could be addressed separately.

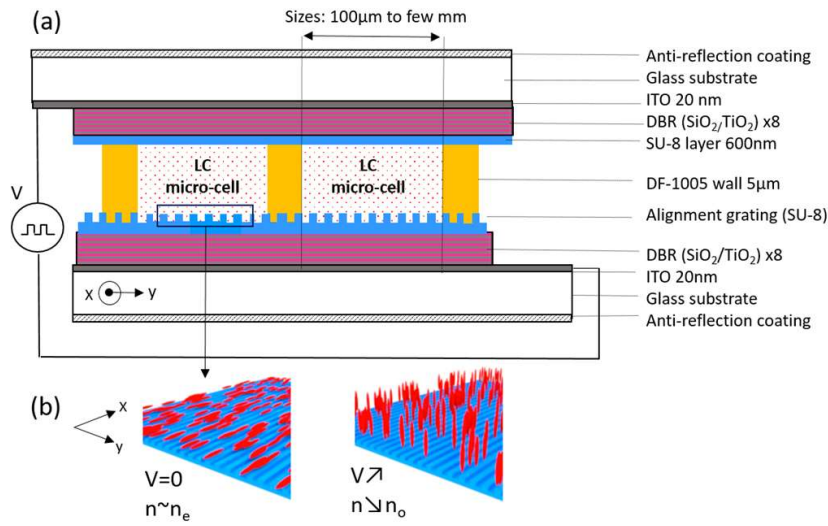

Fig. 1. (a). Schematic of tunable optical filter incorporating a liquid crystal microcell and a nanoimprinted alignment grating. (b) Schematic of alignment behavior of LC along the grating as a function of square wave AC voltage applied to the LC microcell.

\section{B. LC alignment grating}

LC alignment is ensured by a single grating that is nanoimprinted on the surface of the bottom mirror. Preliminary tests by assembling LC macro-cells demonstrated indeed that efficient LC anchoring and alignment were obtained with only one alignment grating. This greatly simplifies the technology and avoids the need of a precise alignment between the two substrates. The choice of the grating period $\Lambda$ must take into account both the detrimental optical diffraction losses and the azimuthal LC anchoring energy magnitude, that is inversely proportional to the cube of the period and proportional to the square of groove depth [15]. Hence, a sub-wavelength period should be chosen. The period $\Lambda$ was set to $800 \mathrm{~nm}$ and the duty cycle was fixed to 50:50, in order to correspond to a pattern size easily achievable using standard projection photolithography. After the photolithography step, the resist patterns were transferred by conventional dry etching ( $\mathrm{ICP} \mathrm{SF}_{6} / \mathrm{C}_{4} \mathrm{~F}_{8} / \mathrm{O}_{2}$ ) into a silicon wafer of 6 " in diameter. The grating depth was chosen in the range [70-80nm] to obtain a sufficient energy for enabling an efficient uniform planar anchoring [16]. A typical atomic force microscopy (AFM) image of the silicon mold surface in shown in Fig. 2. This hard mold was then transferred by thermal-NIL in a second one, made of a thermo-formable material $\left(\right.$ Zeonor $\left.^{\mathbb{B}}\right)$, easier to implement whatever the destination substrate's topography (Fig. 2(a)).
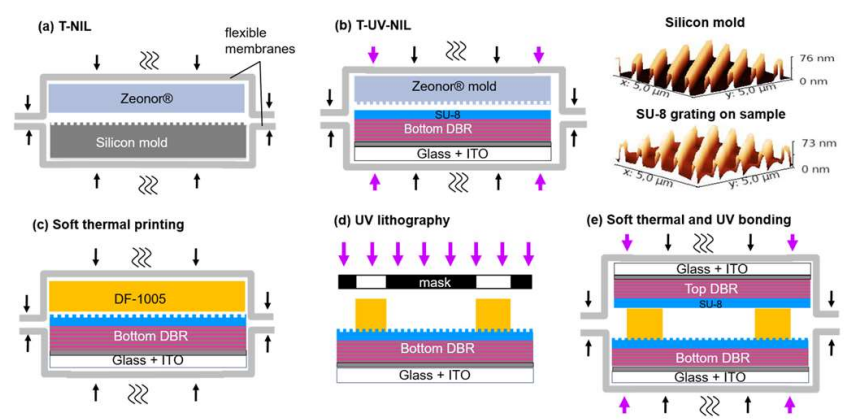

Fig. 2. Process steps: (a) Thermal NIL of the grating with Si mold in a Zeonor ${ }^{\circledR}$ film $\left(\mathrm{T}=145^{\circ} \mathrm{C}, \mathrm{N}_{2}\right.$ pressure $=31$ bars, $\left.3 \mathrm{~min}\right)(\mathrm{b})$ Thermal-UV NIL in a SU-8 layer on top DBR with Zeonor ${ }^{\circledR}$ soft mold $\left(\mathrm{T}=80^{\circ} \mathrm{C}, \mathrm{N}_{2}\right.$ pressure $=13$ bars, 2 min) and AFM images of the surfaces of the Si mold and of the SU-8 grating on the sample (area $5 \times 5 \mu \mathrm{m}^{2}$ ) (c) Soft thermal printing of DF-1005 film (T= $40^{\circ} \mathrm{C}, \mathrm{N}_{2}$ pressure $=0.7$ bars, $1 \mathrm{~min}$ ) (d) Definition of DF-1005 walls (e) Soft thermal and UV bonding of the top mirror using a second SU-8 layer ( $\mathrm{T}=120$ ${ }^{\circ} \mathrm{C}, \mathrm{N}_{2}$ pressure $=5$ bars, $2 \mathrm{~min}$ ). No alignment is required between two steps.

\section{LC-microcell tunable filter fabrication}

The first step of microcell fabrication consists of spin-coating a $600 \mathrm{~nm}$-thick SU-8 layer on the bottom mirror's surface. The alignment grating is then imprinted in this layer using the above-described soft mold (Fig. 2(b)). As seen in AFM images, a good transfer of the grating patterns in the SU-8 resist is obtained. LC microcells are then defined by polymer walls made of an epoxy photosensitive film named DF-1005. DF1000 series are dry thick resist films that present similar optical and mechanical properties than $\mathrm{SU}-8$, while being easier to implement, of lower cost, and having a better thickness reproducibility than spin-coated $\mathrm{SU}-8$ whatever the substrate 
shape and size ( $4.8 \pm 0.2 \mu \mathrm{m}$ for DF-1005 films) [17]. The DF1005 film is printed on the mirror's surface by soft thermal transfer (Fig.2(c))[18]. It is worth noting that the NIL setup we used is equipped with a double-sided flexible chamber that ensures an isostatic pressure on the wafer (Nanonex NX-2500) [12]. This constitutes a great advantage to handle fragile samples such as VCSEL ones. The DF-1005 walls of the cells are defined by standard photolithography (with lateral sizes than can range from few $\mu \mathrm{m}$ to few $\mathrm{mm}$ ) followed by a standard development and a hard bake (Fig.2(d)). The top mirror, covered by the same SU-8 layer as the bottom mirror, is subsequently bonded by thermal-UV-NIL onto the DF-based walls of the first substrate to form the polymer microcells. Microcell sealing is then achieved by UV exposure of the SU8 layer through the UV-transparent top mirror (Fig.2(e)). A top view of a fabricated sample is shown in the inset of Fig. 6. Finally, the microcells are filled under vacuum with LC (E7), at a temperature higher than its nematic-isotropic transition (i.e. $>70^{\circ} \mathrm{C}$ ) thanks to lateral apertures on the sample. At the end of the process, the filter arrays consists in several microcells, from circular areas of $800 \mu \mathrm{m}$ in diameter to square surfaces up to $3 \times 3 \mathrm{~mm}^{2}$. Figure 3 shows an optical microscope image of the filled cells observed by transmission microscopy between two crossed polarizers. When the sample orientation is at $45^{\circ}$ relatively to the polarizers axis, an uniform color can be observed in all cells, as seen in Fig. 3(a). This proves that a uniform thickness of LC layer is ensured on the whole surface. Rotating the wafer, $\mathrm{LC}$ director is then aligned to one polarizer axis and a total extinction is obtained, as shown in Fig. 3(b). This demonstrates the good efficiency of LC alignment process on the whole sample surface.
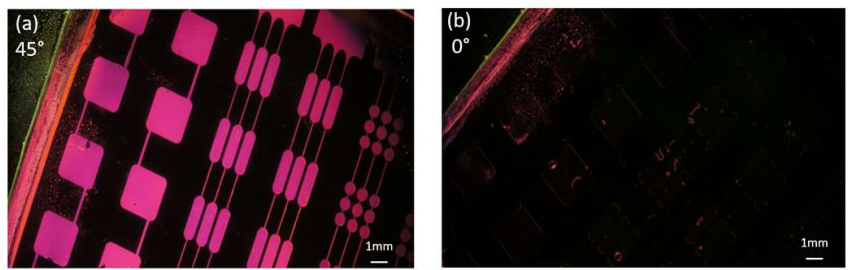

Fig. 3. Microscope views of LC microcells between crossed polarizers for different angular orientations of the sample. (a) sample axis at $45^{\circ}$ to polarizer axis: LC microcells are visible and have a uniform color. (b) LC director aligned with the polarizer axis $\left(0^{\circ}\right)$ : total extinction

\section{RESULTS AND DISCUSSION}

\section{A. Filter bandwidth}

The micro-cell filter bandwidth was first determined with a transmission set-up using a narrow-band $(\sim 50 \mathrm{~nm})$ fibered, nonpolarized Superluminescent Light Emitting Diode (SLED) as the optical source. The collimated output of the SLED was focused under normal incidence onto the sample fixed to a XYZ translation stage and positioned between two optical lenses in a confocal configuration. The focused spot diameter was of $\sim 80$ $\mu \mathrm{m}$. The transmitted light was then directed to either an optical spectrum analyzer with a spectral resolution of $0.02 \mathrm{~nm}$, or a photodiode monitoring the total transmitted power.

Figure 4 shows the transmitted spectrum of an unbiased microcell and the comparison with the modeling. Considering the dispersion properties of the different layers, determined by ellipsometry and transmission measurements, and a $5 \mu \mathrm{m}$ thick microcell filled with E7, the theoretical filter free spectral range (FSR) for each polarization and filter linewidth are calculated to be of $\sim 100 \mathrm{~nm}$, and $0.30 \mathrm{~nm}$, respectively, in the absence of any optical loss. The spectral separation between the ordinary and extraordinary modes without applied voltage is expected to be of $\sim 20 \mathrm{~nm}$, and the maximum tuning range for the extraordinary mode is $\sim 150 \mathrm{~nm}$ (actually limited by the FSR in our design). The experimental spectral spacing between the two orthogonally-polarized resonant modes is $23 \mathrm{~nm}$ and is well reproduced if one considers a refractive index $\mathrm{n}_{0}=1.507$ at $1.55 \mu \mathrm{m}$ [14] for the ordinary mode, and $\mathrm{n}=1.696$ for the "extraordinary" mode at $0 \mathrm{~V}$. This value is very close to the extraordinary index value $\mathrm{n}_{\mathrm{e}} \sim 1.697$, confirming that the alignment is planar. The $-3 \mathrm{~dB}$ filter bandwidth is measured to be of $\sim 0.50 \mathrm{~nm}$ for the ordinary mode and $\sim 0.35 \mathrm{~nm}$ for the extraordinary mode, close to the theoretical values. Finally, the absolute peak transmission for both the ordinary and "extraordinary" modes has been measured to be in the range of $57 \%$ to $63 \%$, corresponding to an insertion loss of $2.0 \mathrm{~dB}$ to 2.2 dB. A polarizer was inserted at the collimated output of the SLED for these measurements. In the absence of loss in the microcell, the theoretical filter insertion loss is due to the absorption in the ITO layers and calculated to be of $\sim 1.4 \mathrm{~dB}$ at $1.55 \mu \mathrm{m}$. Considering the high value of the peak-transmittance, the small broadening of the measured transmitted spectrum may be accounted for by some inhomogeneity of the microcell thickness (by at most 2-3nm) or by some residual nonuniformity of the LC alignment direction over the probed area.

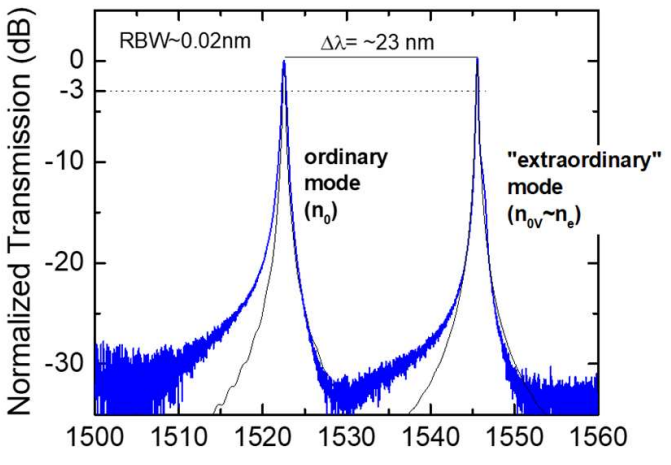

Wavelength $(\mathrm{nm})$

Fig. 4. Measured spectrum of the transmitted signal (blue line), and comparison with modeling (black line). In the calculations, the thicknesses of the SU-8 and LC (E7) layers are fixed to $605 \mathrm{~nm}$ and $4800 \mathrm{~nm}$, respectively and the intracavity absorption losses are assumed to be zero, except for the ITO layers which are placed outside the cavity.

\section{B. Tuning range}

The broadband tunability of the micro-filters was characterized using a Fourier transform infra-red spectrometer (FTIR) equipped with a microscope stage and two polarizers. This made it possible to measure the polarization-resolved reflectivity spectrum of a microcell in a small area $(\sim 100 \mu \mathrm{m})$ over a wide spectral range. Figure 5 shows a $3 \mathrm{D}$ color map of the reflectivity spectra measured for applied peak voltages varying from 0 to $18 \mathrm{~V}(20 \mathrm{kHz})$. The FSR measured between two successive extraordinary modes is $102 \mathrm{~nm}$, which is in good agreement with our design. The probed cell area was slightly 
different from the one previously measured with the SLED setup, which explains the $0.6 \%$ difference of the extraordinary mode wavelength at $0 \mathrm{~V}$ bias. Looking at the evolution of the mode centered near $1.55 \mu \mathrm{m}$, one can see that the tuning starts at a threshold voltage of $3 \mathrm{~V}$ and that the tuning range is equal to the FSR $(102 \mathrm{~nm})$ when the applied voltage reaches $18 \mathrm{~V}$. The corresponding tuning ratio $\left(\Delta \lambda / \lambda_{0}\right)[1]$ is equal to $6.5 \%$, thus demonstrating the potentialities of LC technology for widelytunable VCSEL fabrication. Moreover, as seen in Figure 6, the maximal variation of the modes peak wavelength is measured to be $3 \%$ across the sample, confirming the high thickness homogeneity of the DF-1005 resist. Finally, the large-scale and in-cell variations of the linewidth are typically comprised between $0.3 \mathrm{~nm}$ and $0.57 \mathrm{~nm}$.

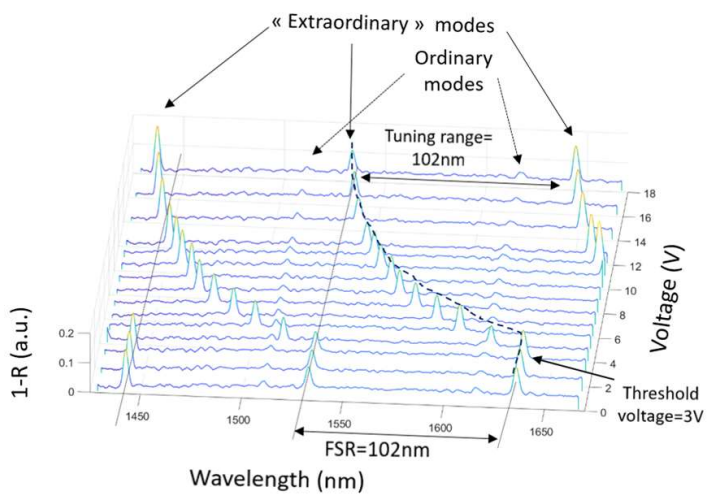

Fig. 5. Evolution of reflectivity spectra (plotted as 1-R for improved clarity) measured by localized FTIR spectroscopy for different AC applied peak voltages $(20 \mathrm{kHz})$ varying from 0 to $18 \mathrm{~V}$ in amplitude. The orientation of the two polarizers was chosen to enhance the "extraordinary" modes. The spectral width of the transmitted peaks is limited by the FTIR nominal resolution set-up $(0.2 \mathrm{~nm})$ and by the numerical aperture of the used microscope objective (N.A=0.1). The tuning voltage threshold is $3 \mathrm{~V}$ and the tuning range near $1.55 \mu \mathrm{m}$ is equal to the FSR $(102 \mathrm{~nm})$.

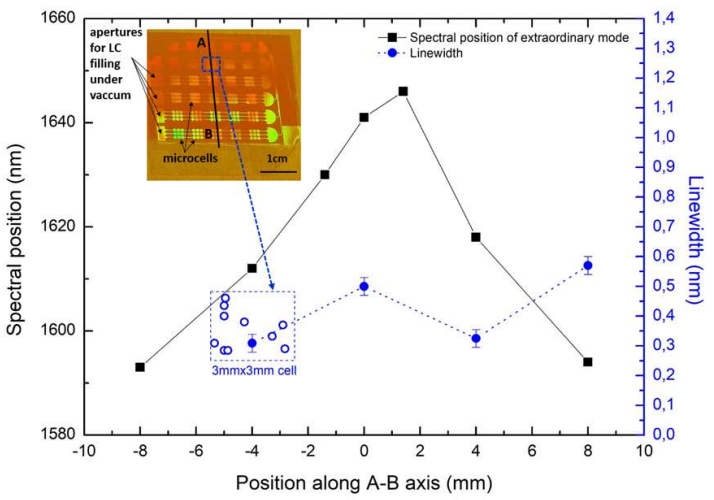

Fig. 6. Evolution of mode spectral position of the extraordinary mode measured along A-B axis across the wafer (black squares) and of mode linewidth across the wafer (blue filled circles) and in a $3 \mathrm{~mm}^{2}$ square cell (blue open circles). Errors bars correspond to the bandwidth resolution of the spectrum analyzer. Inset: top view of a fabricated sample $\left(4 \times 4 \mathrm{~cm}^{2}\right)$ with position of A-B axis.

\section{CONCLUSIONS}

We have demonstrated a wafer-scale LC microcell technology which enables the collective fabrication of high finesse tunable optical micro-filters. A tuning range as wide as $100 \mathrm{~nm}$ was obtained in the $1550 \mathrm{~nm}$-band with small operating voltage $(18 \mathrm{~V})$ and no current consumption. The filter insertion loss was limited to $\sim 2 \mathrm{~dB}$. Furthermore, the measured narrow bandwidth $(0.35 \mathrm{~nm})$ in very good agreement with simulations demonstrated that such microcells arrays present negligible internal losses and could be integrated at a wafer-scale into active optoelectronic devices such as CWDM photodetectors or tunable VCSELs.

\section{REFERENCES}

[1] P. Qiao, K. Cook, K. Li, and C. J. Chang-Hasnain, "Wavelength swept VCSELs", IEEE J. Sel. Top Quantum Electron. 23, 1700516, (2017)

[2] J. S. Patel, M. A. Saifi, D. W. Berreman, Chinlon Lin, N. Andreadakis, and S. D. Lee "Electrically tunable optical filter for infrared wavelength using liquid crystals in a Fabry-Perot étalon", Appl. Phys. Lett. 57, 1718, (1990).

[3] M. W. Maeda, J. S. Patel, C. Lin, J. Horrobin and R. Spicer, "Electronically tunable liquid-crystal-etalon filter for high-density WDM systems," in IEEE Photonics Technology Letters, vol. 2, no. 11, pp. 820-822, Nov. 1990.

[4] K. Hirabayashi, H. Tsuda and T. Kurokawa, "Narrow-band tunable wavelength-selective filters of Fabry-Perot interferometers with a liquid crystal intracavity," in IEEE Photonics Technology Letters, vol. 3, no. 3, pp. 213-215, March 1991

[5] Y.-H. Yao, C.-T. Wang, R.-R. Chen, H.-C Jau, Y.-J. Chiu, and T.H. Lin, "Wavelength tunable infrared light source based on semiconductor-integrated liquid crystal filter," Opt. Express 20, 22872-22877 (2012).

[6] Y. Xie, J. Beeckman, K. Panajotov, and K. Neyts, "Vertical-cavity surface-emitting laser with a liquid crystal external cavity," Opt. Lett. 39, 6494-6497 (2014).

[7] C. Belmonte, L. Frasunkiewicz, T. Czyszanowski, H. Thienpont, J. Beeckman, K. Neyts, and K. Panajotov, "Optimization of electrically tunable VCSEL with intracavity nematic liquid crystal," Opt. Express 23, 15706-15715 (2015).

[8] O. Castany, L. Dupont, A. Shuaib, J. P. Gauthier, C. Levallois, and C. Paranthoën "Tunable semiconductor vertical-cavity surfaceemitting laser with an intracavity liquid crystal layer"Appl. Phys. Lett. 98, 161105 (2011)

[9] R. Beccherelli, I.G. Manolis and A. d'Alessandro, "Characterisation of photoalignment materials for photonic applications at visible and infrared wavelengths Mol. Cryst. Liq. Cryst. 429 227-35, 2005.

[10] O. Castany and L. Dupont, "Collective constructions with microbricks. Application to the fabrication of liquid crystal micro-cells", J. Micromech. Microeng. 22 125009, 2012.

[11] D. C. Flanders, D. C. Shaver, and Henry I. Smith, "Alignment of liquid crystals using submicrometer periodicity gratings" Appl. Phys. Lett. 32, 597 (1978).

[12] S. P. Chang, K. J. Morton, H. Tan, P. F. Murphy, W. Wu and S. Y. Chou, "Tunable Liquid Crystal-Resonant Grating Filter Fabricated by Nanoimprint Lithography," in IEEE Photonics Technology Letters, vol. 19, no. 19, pp. 1457-1459, Oct.1, 2007.

[13] P. G. de Gennes, J. Prost "The Physics of Liquid Crystals", Clarendon Press, 27 avr. 1995

[14] J. Li, S.T. Wu, S. Brugioni, R. Meucci, R., and S. Faetti, (2005). Infrared refractive indices of liquid crystals. Journal of Applied Physics, 97(7), 073501

[15] C.Gear, K. Diest, V. Liberman, and M. Rothschild, "Engineered liquid crystal anchoring energies with nanopatterned surfaces," Opt. Express 23, 807-814 (2015)

[16] D. W. Berreman "Solid Surface Shape and the Alignment of an Adjacent Nematic Liquid Crystal” Phys. Rev. Lett. 28, 1683 -26 June 1972;

[17] http://emsadhesives.com/

[18] S Abada, L. Salvi, R. Courson, E. Daran, B. Reig, J.B. Doucet, T. Camps and V. Bardinal, "Comparison of lamination and soft thermal printing of dry thick photoresist films for the uniform fabrication of polymer MOEMS on small-sized samples" J. Micromech. Microeng. 27 055018, 2017. 